Disponível em

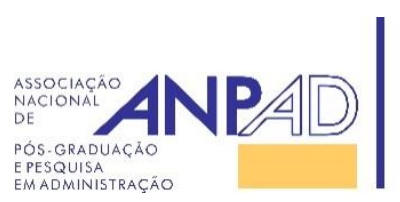

http://www.anpad.org.br/rac

\title{
Técnicas de Prospecção: Um Estudo Comparativo
}

Forecast Techniques: A Comparative Study

Dálcio Roberto dos Reis ${ }^{1}$ Ticiana Braga de Vincenzi ${ }^{1}$ Fabricio Palermo Pupo ${ }^{1}$

Universidade Positivo ${ }^{1}$ 


\title{
Resumo
}

As tecnologias futuras podem afetar o negócio da empresa, e sua prospecção é um passo importante no planejamento, especialmente das empresas que atuam em tecnologias de ponta. O objetivo deste trabalho é realizar um estudo teórico comparativo sobre as principais técnicas de prospecção de tecnologias futuras, avaliadas em relação a seis critérios: custo para operacionalização; número necessário de pessoas; tempo necessário para obter o resultado; complexidade da operacionalização, ou seja, facilidade de aplicação; tipo de análise (subjetiva ou objetiva), sendo as técnicas quantitativas consideradas como mais objetivas; confiabilidade dos resultados (consistência e replicabilidade). Com esse estudo comparativo, são fornecidos parâmetros para ajudar as empresas na seleção das técnicas prospectivas mais adequadas, de acordo com suas necessidades. Em termos metodológicos, é uma pesquisa de natureza qualitativa. Quanto ao propósito, constitui uma pesquisa descritiva que utiliza levantamento bibliográfico e pesquisa documental como métodos de coleta de dados. Conclui-se que é vantajoso usar várias técnicas simultaneamente, proporcionando mais eficiência e fazendo com que as previsões sejam mais robustas. Por fim, espera-se que este artigo inicie um debate sobre os critérios de seleção de técnicas de prospecção de tecnologias futuras.

Palavras-chave: métodos e técnicas de prospecção; cenários; forecast; estudos do futuro.

\begin{abstract}
Future technologies may affect a company's business, and prediction of such technologies is an important step in the planning process, especially regarding cutting-edge technology industries. The objective of this paper is to develop a comparative theoretical study on key techniques aimed at predicting future technologies, evaluated according to six criteria: operation costs; required number of people; required time to obtain results; operation complexity, that is, ease of implementing techniques; analysis type (subjective or objective), wherein quantitative techniques are considered more objective; and reliability of results (consistency and replicability). Thus, this study provides parameters to assist companies in selecting the most suitable prospective techniques, according to their needs. The research methodology is qualitative, and the study scope is a descriptive analysis using bibliographic and documentary research as data collection methods. The conclusion is that it is advantageous to use several methods simultaneously, providing higher efficiency and producing better assessments. Finally, it is hoped that this article launches a debate on the assigned criteria for techniques used to predict future technologies.
\end{abstract}

Key words: predictive methods and techniques; scenarios; forecast; future studies. 


\section{Introdução}

Em seu livro Administrando em Tempos de Grandes Mudanças, Peter Drucker (1996) afirma que não é difícil nem importante prever o futuro. Entretanto, no que diz respeito às inovações tecnológicas, é melhor a empresa estar ciente dos rumos do mercado e preparada para enfrentar ou aproveitar essas novas tecnologias (Reis \& Lobo, 2015).

A forma usual pela qual as empresas se preparam para o futuro é realizando planejamento estratégico. O planejamento estratégico se divide nas seguintes etapas (Kotler \& Keller, 2006): definição da missão do negócio; análise interna, para avaliar as forças e os pontos fracos da empresa; análise externa, para avaliar oportunidades e ameaças de mercado; definição dos principais objetivos da empresa e das ações para atingi-los; implantação do plano; por fim, acompanhamento do planejado em relação ao realizado.

Existe um passo no processo de planejamento, inserido na etapa de análise externa, que é fundamental para a sobrevivência no longo prazo, principalmente para companhias que trabalham com tecnologia de ponta: a prospecção de novas tecnologias que podem alterar o negócio da organização. $\mathrm{O}$ objetivo deste trabalho, portanto, é fazer um estudo teórico comparativo sobre as principais técnicas de prospecção de tecnologia existentes, apresentando as vantagens e dificuldades de cada técnica, assim como em que situações devem ser utilizadas. Justifica-se este artigo pelo apoio a empresas e instituições na identificação e na escolha de qual a melhor técnica de acordo com suas necessidades.

\section{Revisão de Literatura}

O objetivo das técnicas de prospecção é criar, explorar e testar os futuros possíveis e desejáveis para melhorar as decisões, incluindo a análise de como essas condições podem mudar a partir da implementação de políticas e ações. A pesquisa pode ser direcionada para questões de pequena ou grande escala, em um futuro próximo ou distante, e pode projetar condições possíveis ou desejadas (Glenn, 2003a). Segundo Miles, Keenan e Kaivo-Oja (2002), o trabalho de prospecção visa a interligar várias forças, tendências e fatores condicionantes, a fim de visualizar futuros alternativos (em vez de prevê-los). Em um ambiente acelerado e dinâmico, o desafio torna-se ainda maior. Por isso a importância e a dificuldade da empreitada das empresas para ativamente monitorar as tendências emergentes, desenvolver cenários alternativos que reflitam as potenciais oportunidades de negócios que possam surgir e enfrentar os desafios do futuro (Battistella \& De Toni, 2011).

A questão não é sobre utilizar ou não técnicas formais de prospecção, mas de que forma e quais técnicas devem ser usadas. Existem vários critérios possíveis para selecionar as técnicas, tais como: disponibilidade de recursos, especialmente tempo e dinheiro; amplitude e profundidade da participação de especialistas; resultados desejados, que podem ser mais ou menos orientados ao processo ou ao produto; requisitos quantitativos e/ou qualitativos, especialmente onde dados não estão disponíveis (Miles \& Keenan, 2003). Na literatura sobre técnicas prospectivas há diversas denominações e terminologias para estruturas conceituais, o que dificulta a identificação de qual recurso é mais adequado para cada situação. Isso faz com que seja usual constatar técnicas desenvolvidas para usos específicos sendo empregadas em temas de natureza ampla e complexa, o que pode levar a resultados pouco confiáveis (Centro de Gestão e Estudos Estratégicos [CGEE], n.d.).

Porter et al. (2004) diferenciam as técnicas de prospecção considerando se são principalmente duras, isto é, quantitativas, empíricas e numéricas, ou suaves, isto é, qualitativas, baseadas em julgamentos e refletindo conhecimentos tácitos. Algumas técnicas se enquadram nas duas definições. As técnicas quantitativas dependem fortemente da representação numérica dos desenvolvimentos possíveis. Elas têm vantagens consideráveis (por exemplo, capacidade de estimar taxas e escalas de mudança), e também desvantagens (simplificação de variáveis políticas e sociais importantes, etc.) 
(Miles \& Keenan, 2003). As técnicas qualitativas são muitas vezes empregadas onde tendências e desenvolvimentos são difíceis de capturar através de indicadores simplificados, ou quando tais dados não estão disponíveis. Várias formas de pensamento criativo são incentivadas pelas abordagens qualitativas, como brainstorming, escrita utópica e ficção científica. Técnicas para trabalhar sistematicamente com dados qualitativos estão se tornando mais disponíveis com o desenvolvimento da tecnologia da informação (Miles \& Keenan, 2003).

Porter et al. (2004) também classificam as técnicas em normativas, cujo processo parte de uma futura necessidade percebida, e em exploratórias, cujo processo começa com a extrapolação dos atuais recursos. As técnicas exploratórias começam com o momento presente como ponto de partida, e avançam para o futuro, seja com base em extrapolação de tendências passadas ou dinâmicas causais, ou então em eventos que podem estar fora das tendências conhecidas. A maioria dos estudos de previsão é exploratória, embora, quando estes resultam em previsões alarmantes, em geral há um esforço para localizar os pontos de inflexão que poderiam criar um futuro mais desejável (Miles \& Keenan, 2003). Técnicas normativas, ao contrário, começam com uma visão preliminar de um possível ou desejável conjunto de futuros que são de particular interesse. Essas técnicas, então, olham para trás para averiguar se esses futuros podem ou não acontecer a partir do presente, e como podem ser alcançados, ou evitados, dados os recursos, as tecnologias e as restrições disponíveis. Um desenvolvimento relativamente recente é o uso de cenários de sucesso e de oficinas, onde os participantes estabelecem uma visão compartilhada de um futuro desejável, e identificam as formas para que possa ser alcançado (Miles \& Keenan, 2003).

Por fim, Porter et al. (2004) diferenciam as técnicas de prospecção em nove famílias distintas. A classificação foi feita de acordo com as características das técnicas, considerando que algumas compilam informações, outras procuram compreender as interações entre os eventos, ou abordam incertezas e envolvem análise probabilística. Assim, as habilidades necessárias tendem a ser diversas. As nove famílias são: opinião de especialistas; cenários; análise de tendências; avaliação e decisão; modelagem e simulação; criatividade; descritivas e matrizes; estatísticas; monitoramento e sistemas de inteligência. Algumas técnicas podem ser classificadas em duas famílias diferentes (Porter et al., 2004).

\section{Metodologia}

Este é um estudo de natureza qualitativa e de corte transversal, pois foi realizada uma análise comparativa das técnicas existentes e em uso nas atividades prospectivas. Quanto ao propósito, constitui uma pesquisa descritiva (Richardson, 1999), feita com levantamento bibliográfico e pesquisa documental descritiva como método de coleta de dados, e com análise documental, para sintetizar e interpretar o conteúdo dos documentos coletados.

Foram usadas fontes secundárias para avaliar os métodos de prospecção, tais como: publicações do Centro de Gestão e Estudos Estratégicos (CGEE - organização supervisionada pelo Ministério da Ciência, Tecnologia e Inovação), do The Millennium Project publicado pela American Council for the United Nations University (Futures Research Methodology), da European Foundation for the Improvement of Living and Working Conditions (Handbook of Knowledge Society Foresight) e diversos outros artigos sobre o tema, citados em cada caso específico.

Para realizar o estudo teórico comparativo sobre as principais técnicas de prospecção em tecnologia existentes, utilizou-se a diferenciação por famílias apresentada por Porter et al. (2004). Os critérios de comparação usados foram: quanto ao custo para operacionalização das técnicas; quanto ao número necessário de pessoas; quanto ao tempo necessário para obter o resultado; quanto à complexidade da operacionalização; quanto ao tipo de análise (subjetiva ou objetiva); quanto à confiabilidade dos resultados. Esses critérios foram escolhidos por terem sido citados isoladamente por autores que estão identificados, na sequência, na análise das diversas famílias. Além disso, em pesquisa realizada por Carbone e Armstrong (1982) com praticantes e acadêmicos, especializados em técnicas de prospecção, sobre os critérios considerados importantes para avaliar essas técnicas, alguns dos critérios 
mais frequentemente citados foram: facilidade de interpretação, custo/tempo, e facilidade de uso e aplicação.

Para cada critério foram atribuídas notas baseadas em comparações entre as famílias de técnicas, de acordo com as características recolhidas na análise bibliográfica. Considerou-se, no critério custo para operacionalização, a quantidade de recursos, tais como ferramentas, sistemas e equipamentos necessários para a realização da família de técnicas, sendo que, quanto menor o custo, maior a nota. Em relação ao número necessário de pessoas, estimou-se a quantidade de participantes em todas as etapas, tanto auxiliando quanto participando diretamente; sendo que, quanto menor a quantidade de pessoas, maior a nota. No tempo necessário para obter o resultado, avaliou-se a duração total do processo, incluindo o tempo de planejamento, a execução e a obtenção dos resultados, sendo que, quanto menor o tempo, maior a nota. No critério complexidade da operacionalização, observou-se a facilidade para colocar a técnica em prática, sendo que, quanto mais complexa, menor a nota. Quanto ao tipo de análise (subjetiva ou objetiva), as técnicas quantitativas, ou "duras" (Porter et al., 2004, p. 289), foram consideradas como as mais objetivas; sendo que, quanto mais objetivas, maior a nota, devido à facilidade de interpretação e aplicação dos resultados. A confiabilidade está relacionada à capacidade de replicabilidade e à consistência dos resultados que uma técnica apresenta (Hammersley, 1992; Kirk \& Miller, 1986; Silverman, 2006). A confiabilidade indica o grau em que um determinado procedimento produz os mesmos resultados em ensaios repetidos com as mesmas ferramentas empíricas ou equivalentes (Corbetta, 2003), sendo que, quanto maior a confiabilidade, maior a nota.

\section{Caracterização das Famílias de Técnicas}

Na família de opinião de especialistas, as principais técnicas são: Delphi (pesquisa interativa); Grupos Focais (painéis e workshops); Entrevistas e Técnicas Participativas (Porter et al., 2004). A técnica Delphi tem como objetivo obter, de um grupo de especialistas, um consenso de opinião o mais confiável possível. A técnica emprega o repetido questionamento individual dos peritos, intercalado com a divulgação controlada das opiniões entre eles (Dalkey \& Helmer, 1963). Os Grupos Focais são geralmente realizados por um pesquisador treinado, que orienta a conversa entre um pequeno grupo de entrevistados (Glenn, 2003b). Técnicas participativas podem envolver um grupo em um mesmo local ou geograficamente e temporalmente disperso, mas ligado por telecomunicações. Outros exemplos são: Pesquisas de Opinião, Charrette, Syncon, VisionQuest, Computer Groupware, etc. (Glenn, 2003b).

Na família de cenários, as principais técnicas são: Field Anomaly Relaxation Methods (FAR); Cenários (com verificações de consistência e Gestão de Cenários); Simulação de Cenários (Gaming e cenários interativos) (Porter et al., 2004). A técnica FAR tem como objetivo proporcionar uma gama de cenários internamente consistentes como contextos para a tomada de decisão, gerando o histórico, e não apenas o resultado final (Coyle, 2003). Um cenário é uma história que conecta descrições de futuro à realidade atual por meio de relações causais que ilustram decisões e suas consequências. Cenários descrevem eventos e como eles tendem a evoluir (Glenn, 2003c).

Na família de análise de tendências, as principais técnicas são: Análise da Onda Longa ou Ciclo Prolongado; Precursor Analysis; Extrapolação de Tendências (montagem e projeção de curva de crescimento); Análise de Tendência de Impacto (Trend Impact Analysis [TIA]) (Porter et al., 2004). A análise de tendências envolve predição por meio da continuação de dados históricos quantitativos. A Onda Longa é caracterizada por sucessivas ondas de expansão e colapso da economia (Sterman, 1985). Linstone (2002) observa que períodos de inovação são seguidos por períodos de consolidação de conhecimentos, e parecem coincidir com as ondas longas. Esses períodos devem ser considerados na elaboração de forecasts. O processo de Precursor Analysis tem como finalidade identificar possíveis fontes de problemas na forma de eventos anômalos que podem sinalizar uma condição potencial significativa de risco no sistema (National Aeronautics and Space Administration [NASA], 2011). A Análise de Tendência de Impacto (TIA) é uma abordagem de previsão em que uma série de tempo é 
modificada para levar em conta as percepções sobre como os eventos futuros podem mudar extrapolações esperadas (Gordon, 2003a).

Na família de avaliação e decisão, as principais técnicas são: Análise de Ação (Opções); Analytical Hierarchy Process (AHP); Análise de Custo-Benefício (monetizada e outras); Análise de Decisão (Análise de Utilidade); Modelagem de Base Econômica (Input-Output Analysis); Árvores de Relevância (Futures Wheel); Análise de Requisitos (Análise das Necessidades; Matriz de Atributo X Tecnologia); Stakeholder Analysis (Policy Capture; Assumptional Analysis) (Porter et al., 2004). O AHP é uma técnica que divide uma situação complexa em variáveis, atribui valores numéricos para julgamentos subjetivos sobre a importância relativa de cada variável e sintetiza os julgamentos para determinar quais variáveis possuem os pesos mais altos e deveriam ser postas em prática (Saaty, 2008). A Análise de Decisão fornece estrutura e orientação para pensar sistematicamente sobre decisões difíceis, provendo compreensão clara do problema (Clemen, 1996). A Árvore de Relevância subdivide um tema amplo em subtópicos. O resultado mostra uma representação pictórica com uma estrutura hierárquica (The Futures Group International, 2003).

$\mathrm{Na}$ família de modelagem e simulação, as principais técnicas são: Modelagem de Agentes; Modelos Causais; Complex Adaptive System Modeling (CAS; Chaos); Cross-Impact Analysis; Diffusion Modeling; Modelagem de Base Econômica (Input-Output Analysis); Simulação de Cenários (jogos e cenários interativos); Análise da Sustentabilidade (Análise do Ciclo de Vida); Simulação de Sistemas (System Dynamics, KSIM); Avaliação de Tecnologia; Substituição Tecnológica (Porter et al., 2004). Um modelo é uma representação simplificada da dinâmica de uma parte do mundo real. Modelagem de Agentes envolve a criação de agentes, por computador, que interagem uns com os outros e com o meio ambiente, de acordo com um conjunto de regras semelhantes às da vida real (Gordon, 2003b). CrossImpact Analysis é uma abordagem analítica para as probabilidades de um item em um conjunto de eventos previstos. A maioria dos eventos está de alguma forma relacionada a outros: esta inter-relação é chamada de impacto cruzado (Gordon, 2003c). Simulação de Sistemas fornece a oportunidade de explorar várias opções para lidar com as situações futuras, permitindo que os participantes decidam como reagir (Rausch \& Catanzaro, 2003). As simulações podem servir de base para jogos. Um jogo é qualquer atividade com um objetivo que coloca um jogador (que pode ser uma pessoa, um grupo, ou vários grupos) em competição contra outros jogadores ou contra padrões (Rausch \& Catanzaro, 2003).

Na família de criatividade, as principais técnicas são: Brainstorming; (Brainwriting; Nominal Group Process [NGP]); Creativity Workshops (Future Workshops); Análise de Ficção Científica; TRIZ e Vision Generation (Porter et al., 2004). Entre os objetivos da família de técnicas de criatividade, tem sido dada certa atenção à disseminação das técnicas que estimulem a criatividade individual e que podem ser aplicadas para o desenvolvimento da criatividade do grupo, devido ao envolvimento de vários profissionais interligados por um objetivo comum. As preocupações da criatividade individual estão entrelaçadas com as preocupações sobre as técnicas de dinâmicas de grupo (Roper et al., 2011).

$\mathrm{Na}$ família de descritivas e matrizes, as principais técnicas são: Analogias; Backcasting; Checklists para Identificação de Impactos; Innovation System Modeling; Mitigation Analyses; Análise Morfológica; Análises de Decisão Multicritério (Data Envelopment Analysis [DEA]); Avaliação por Múltiplas Perspectivas; Análise Organizacional; Árvores de Relevância (Futures Wheel); Análise de Requisitos (Análise das Necessidades; Matriz de Atributo X Tecnologia); Análise de Risco; Roadmapping (Product-technology Roadmapping); Avaliação de Impacto Social; Stakeholder Analysis (Policy Capture; Assumptional Analysis); State of the Future Index (SOFI); Análise da Sustentabilidade (Análise do Ciclo de Vida); Substituição Tecnológica (Porter et al., 2004). Em seu contexto mais amplo, essas técnicas oferecem uma visão de consenso ou visão de futuro por meio de um mapa da tecnologia disponível para os tomadores de decisão. Uma vantagem importante no uso desse tipo de abordagem é que ela reduz a dependência da crença em modelos e em análise teórica enganosa na tomada de decisões. Mais importante, ela preenche a lacuna entre o previsor, o analista e o modelador, bem como o mundo real do gerente (Linstone, 2003).

Na família de estatísticas, as principais técnicas são: Bibliometria (Research Profiling; Patent Analysis; Text Mining); Análise de Correlação; Cross-Impact Analysis; Análise de Risco; Análise de 
Tendência de Impacto (Trend Impact Analysis [TIA]) (Porter et al., 2004). As técnicas estatísticas buscam entender os elementos que influem sobre determinado fenômeno, pelo relacionamento entre variáveis independentes e a variável dependente que se quer prever. São testados modelos simples de ajuste (linear, exponencial, quadrado ou cúbico) para a variável dependente, a fim de definir os parâmetros do modelo e minimizar o erro. Os modelos econométricos e os não lineares utilizam equações mais complexas (CGEE, n.d.).

Na família de monitoramento e sistemas de inteligência, as principais técnicas são: Bibliometria (Research Profiling; Patent Analysis; Text Mining) e Monitoramento (Environmental Scanning; Technology Watch) (Porter et al., 2004). Monitoramento e suas variações são adequados para tomar conhecimento de mudanças que podem afetar a penetração das tecnologias. Estas técnicas constituem fontes de informações relevantes, pois se mantêm em constante observação e atualização em relação a uma área de interesse e dentro de uma finalidade específica. Algumas análises resultantes destas técnicas podem: expor uma determinada tecnologia emergente, apontar para quem a está desenvolvendo, oferecer um mapeamento de como os subtemas se inter-relacionam e produzir análises de publicações, de patentes ou de citações (Coates et al., 2001). A utilidade do Monitoramento na tomada de decisão vai além das práticas de previsão. Essas técnicas desempenham um papel importante em atividades, tais como seleção de tecnologias, análise de ambientes competitivos e acompanhamento de tendências no desenvolvimento tecnológico (Roper et al., 2011).

\section{Análise Comparativa das Técnicas}

A análise comparativa foi feita com base nas características das principais técnicas de cada família e considerando as diferenças entre as famílias em relação a cada critério. No final da análise, em cada critério, as famílias foram comparadas entre si, e assim foram atribuídas notas de acordo com o desempenho das famílias no critério avaliado.

As notas foram atribuídas em uma escala de 1 a 5 , sendo 5 ótimo, 4 bom, 3 regular, 2 ruim e 1 péssimo. Esse julgamento foi baseado em comparações entre as famílias de técnicas feitas de acordo com as características apontadas por cada autor ao analisar a técnica específica e que estão apresentadas na análise bibliográfica. No que tange aos critérios custo, número de pessoas, tempo e complexidade, quanto menores por família, maiores as notas. Em relação à confiabilidade, quanto maior por família, maior a nota. Já para o tipo de análise, quanto mais objetiva, maior a nota.

\section{Critério: custo para operacionalização das técnicas}

Na família de opinião de especialistas, com o advento de televisão por satélite, videoconferência, fax e internet, especialistas em qualquer lugar do mundo podem facilmente participar. Sistemas colaborativos globalmente dispersos através da internet permitem flexibilidade e menor custo por usuário do que sistemas de grupos fixos (Glenn, 2003b). Já técnicas como Grupos Focais obrigam a presença dos participantes em um mesmo local, além de serem demoradas, demandando facilitadores altamente qualificados, o que as torna, portanto, caras. Por fim, algumas técnicas, como o Syncon, requerem mais recursos financeiros e habilidade do que outros processos e, por isso, são mais difíceis de realizar e replicar (Glenn, 2003b).

Nas técnicas de cenários, Russell Rhyne (1995 como citado em Coyle, 2003), criador da FAR, afirma que um ciclo completo de FAR leva pelo menos 100 ou mais pessoas-semanas de esforço, dada a importância das questões estratégicas abordadas. No entanto, poucas organizações estão dispostas a se comprometer com esse grau de despesa para resultados que não estarão disponíveis no curto prazo.

O custo para operacionalização das técnicas de análise de tendências vai variar de acordo com a quantidade de recursos, isto é, pessoas e equipamentos que serão utilizados para rodar os processos. Mas não são necessárias muitas pessoas para elaborar as premissas iniciais, e um computador comum pode 
processar as curvas de acordo com os dados históricos. Na família de técnicas de avaliação e decisão, a AHP integra dados concretos com julgamentos subjetivos sobre fatores intangíveis e realiza análise de sensibilidade e revisão a um baixo custo, reforçando a capacidade dos gestores na tomada de decisão (Saaty, 2008).

Em relação ao custo das técnicas de modelagem e simulação, computadores trouxeram uma mudança ainda maior nos tipos de modelos e simulações que são utilizados para diferentes fins. Os objetivos (o que a simulação deve realizar) determinam o escopo do projeto, que é essencial para estimar o número necessário de horas de trabalho de cada habilidade profissional para completar a simulação. Uma das vantagens de simulações e jogos é que eles podem ser desenvolvidos em etapas, quando a situação, incluindo restrições de orçamento e de custo-efetividade, exige um projeto gradual (Rausch \& Catanzaro, 2003).

As técnicas de criatividade em geral não envolvem grandes custos, mas há exceções, como se observa na técnica TRIZ, cuja primeira utilização abrangeu o exame de milhares de patentes para depreender padrões de inovação tecnológica e presumir leis de evolução do sistema de tecnologia (Coates et al., 2001). Os custos para operacionalização podem ser considerados relevantes para a aplicação das técnicas descritivas e matrizes, pois dependem de sistemas sofisticados (softwares).

Nas técnicas estatísticas, por serem procedimentos de análises probabilísticas e de frequências, há o uso de softwares licenciados de acordo com as técnicas. Isto faz com que o custo esteja presente, porém, não há comparação de valores entre as técnicas, pois estas têm diferentes objetivos. Na família técnicas estatísticas, há algumas mais populares, como Correlação e Bibliometria. O custo das técnicas de monitoramento e sistemas de inteligência é alto devido aos diversos recursos a serem utilizados para a implementação das técnicas, que tendem a ser dinâmicas e constantes, e nas quais é necessário acesso contínuo a informações muitas vezes privilegiadas e privadas.

A partir da análise das famílias, foram atribuídas as seguintes notas neste critério, apresentadas na Tabela 1: as técnicas de monitoramento e sistemas receberam a menor nota (1) por serem as que apresentam maiores custos; as técnicas de avaliação e decisão receberam a maior nota (5) por serem as de menores custos; em seguida, análise de tendências recebeu nota 4; criatividade, nota 3; as demais famílias receberam nota 2 .

Tabela 1

Avaliação por Família no Critério Custo para Operacionalização das Técnicas

\begin{tabular}{cccccccccc}
\hline Critérios & $\begin{array}{c}\text { Opinião } \\
\text { especial. }\end{array}$ & Cenários & $\begin{array}{c}\text { Análise } \\
\text { tend. }\end{array}$ & $\begin{array}{c}\text { Aval. e } \\
\text { decisão }\end{array}$ & $\begin{array}{c}\text { Model. e } \\
\text { simul. }\end{array}$ & Criatividade & $\begin{array}{c}\text { Descrit. } \\
\text { matrizes }\end{array}$ & Estatísticas & $\begin{array}{c}\text { Monit. e } \\
\text { sistemas }\end{array}$ \\
\hline Custo & 2 & 2 & 4 & 5 & 2 & 3 & 2 & 2 & 1 \\
\hline
\end{tabular}

Nota. Fonte: elaborada pelos autores.

\section{Critério: número necessário de pessoas}

Quanto ao número necessário de pessoas, as técnicas de opinião de especialistas podem variar bastante: desde pequenos grupos de 8 a 12 pessoas reunidas em Grupos Focais, até milhares de especialistas consultados por meio de Pesquisas de Opinião (Glenn, 2003b).

Na família de cenários, segundo Coyle (2003), estima-se que um ciclo de FAR necessita de um ou dois analistas em tempo integral, uma equipe de cerca de quatro pessoas envolvidas diretamente no problema trabalhando metade do dia, e um grupo sênior de supervisão participando quando for preciso. Para a técnica de Cenários, uma equipe é necessária para descrever eventos, tendências e incertezas que podem afetar o processo de tomada de decisões, analisar as forças que irão moldar o ambiente futuro de negócios, e desenvolver teorias de cenários ou lógicas, que são visões diferentes do modo como o mundo poderia funcionar no futuro (Glenn, 2003c). 
Considerando a família de análise de tendências, na Precursor Analysis há algumas etapas de equipe envolvendo especialistas de análise precursora e engenheiros com conhecimento do sistema em estudo. Na aplicação, o número de participantes pode variar. Mas, na generalização de anomalias de sistemas complexos, muitas vezes é benéfico ter vários engenheiros peritos em diferentes subsistemas (NASA, 2011). Na técnica TIA, traça-se uma curva com dados históricos para calcular a tendência vindoura. Em seguida, pareceres de peritos são usados para identificar eventos futuros que poderiam provocar desvios, a probabilidade de ocorrência e seus efeitos esperados na tendência futura (Gordon, 2003a).

Na família de avaliação e decisão, a técnica AHP é adaptável a grupos e indivíduos. Ela fornece uma estrutura lógica para a participação do grupo na tomada de decisão ou na resolução de problemas (Saaty, 2008). Nas técnicas de modelagem e simulação, quanto ao número necessário de pessoas, a utilização de Modelagem de Agentes baseia-se na capacidade do analista em realizar testes de sensibilidade e em apresentar a evolução da simulação na tela. É fácil elaborar programas de agente para quem tem certa qualificação em programação de computadores (Gordon, 2003b). Na técnica CrossImpact Analysis, em geral, a estimativa é realizada por grupos de especialistas de diversas disciplinas abrangidas pelos eventos (Gordon, 2003c). Uma Simulação pode ser executada por apenas uma pessoa ou um número muito pequeno de pessoas, ou ter muitas equipes trabalhando simultaneamente em grandes grupos em locais variados (Rausch \& Catanzaro, 2003).

Geralmente as técnicas de criatividade são feitas em grupos, como é o caso do Brainstorming, que é uma técnica de trabalho em equipe (pode variar de duas a dez pessoas, ou quantas forem convenientes) na qual a intenção é produzir o maior número possível de soluções para um dado problema. Serve para instigar a imaginação e fazer brotar ideias. Os membros de um grupo são convidados a ponderar sobre um problema ou tema (CGEE, n.d.).

Não é possível determinar uma quantidade de pessoas para as atividades da família de técnicas descritivas e matrizes. No entanto, é importante frisar que, assim como em outras técnicas, dependem da existência de especialistas, de séries de dados confiáveis organizados e de estruturas para a compreensão da modelagem de dados (CGEE, n.d.). Em relação ao número necessário de pessoas para as técnicas estatísticas, não é relevante fazer uma análise baseada neste critério, pois se trata de técnicas quantitativas especializadas. O número preciso para se trabalhar pode diferir dependendo da quantidade de objetivos propostos e do tempo esperado, o que também ocorre nas técnicas de monitoramento e sistemas de inteligência.

A partir da análise das famílias, foram atribuídas as seguintes notas neste critério, apresentadas na Tabela 2: as técnicas de avaliação e decisão receberam a maior nota (5) por serem as que necessitam de menos pessoas; em seguida, análise de tendências, cenários, modelagem e simulação, opinião de especialistas e criatividade receberam nota 3; as demais famílias receberam nota 2.

Tabela 2

Avaliação por Família no Critério Número Necessário de Pessoas

\begin{tabular}{ccccccccc}
\hline Critérios & $\begin{array}{c}\text { Opinião } \\
\text { especial. }\end{array}$ & Cenários & $\begin{array}{c}\text { Análise } \\
\text { tend. }\end{array}$ & $\begin{array}{c}\text { Aval. e } \\
\text { decisão }\end{array}$ & $\begin{array}{c}\text { Model. e } \\
\text { simul. }\end{array}$ & Criatividade & $\begin{array}{c}\text { Descrit. Estatísticas } \\
\text { matrizes }\end{array}$ & $\begin{array}{c}\text { Monit. e } \\
\text { sistemas }\end{array}$ \\
\hline $\begin{array}{c}\text { Núm. } \\
\text { pessoas }\end{array}$ & 3 & 3 & 3 & 5 & 3 & 3 & 2 & 2 \\
\hline
\end{tabular}

Nota. Fonte: elaborada pelos autores.

\section{Critério: tempo necessário para obter o resultado}

Quanto ao tempo necessário, as técnicas de opinião de especialistas variam bastante: desde um dia a duas semanas na técnica Charrette (Glenn, 2003b), até três a quatro meses, na técnica Delphi (Gordon, 2003d). Nas técnicas de cenários, o ciclo FAR pode ser feito em cerca de 20 semanas de 
trabalho, com um segundo ciclo, se preciso, exigindo um pouco menos de tempo. Se dois ciclos forem planejados desde o início, porém com recursos limitados, é melhor realizar o primeiro de forma rápida e dedicar a maior parte do tempo no segundo, aprofundando as percepções, intuições e apreciações do primeiro (Coyle, 2003).

Na família de análise de tendências, o processo de Precursor Analysis funciona bem quando realizado em aproximadamente seis meses a um ano após a mais recente anomalia no conjunto de dados (NASA, 2011). Nas técnicas de avaliação e decisão, a fim de determinar um problema complexo e aprimorar juízos de valor, o AHP deve ser repetido de forma progressiva, ou iterado, ao longo do tempo, pois dificilmente se podem esperar soluções imediatas para problemas complicados com os quais se lida por um longo tempo. Cada iteração do AHP constitui um processo de elaboração de hipótese e testes, e o refinamento progressivo das hipóteses leva a um melhor entendimento do sistema (Saaty, 2008).

Na família de modelagem e simulação, como a maioria das técnicas utiliza computadores para processar as análises e gerar os resultados, a duração do processo vai depender da disponibilidade e da quantidade de analistas de sistemas para desenvolver as simulações e alimentar os dados. A partir daí, as análises das respostas obtidas poderão ser realizadas pelos gestores do processo.

No que tange ao tempo necessário para obter o resultado, as técnicas de criatividade podem variar de horas a dias, dependendo dos objetivos propostos ou problemas a serem trabalhados. Não há uma determinante que oriente o período necessário para técnicas descritivas e matrizes. No entanto, não são técnicas de rápido acesso e uso, pois dependem da busca de informações relevantes e condizentes com os objetivos propostos.

O tempo necessário pode variar para as técnicas estatísticas, pois depende dos dados coletados e da forma pela qual são feitas a análise (software) e as inferências. O maior tempo é gasto em coleta e tabulação dos dados, já que o processamento nos programas adequados é rápido. Não há um padrão de tempo necessário nas técnicas de monitoramento e sistemas de inteligência. O Monitoramento pode ser feito durante o período que os gestores considerarem necessário. A Bibliometria ajuda a explorar, organizar e analisar grandes quantidades de dados históricos, permitindo a identificação de padrões não aparentes que podem colaborar no processo de tomada de decisão (Daim, Rueda, Martin, \& Gerdsri, 2006).

A partir da análise das técnicas, foram atribuídas as seguintes notas neste critério, apresentadas na Tabela 3: as técnicas de criatividade receberam nota 5 por serem as que apresentam, relativamente às outras, menor tempo; as famílias de avaliação e decisão e opinião de especialistas receberam nota 4; as de monitoramento e sistemas, modelagem e simulação, descritivas e matrizes e estatísticas, nota 3; as técnicas de cenários receberam nota 2 ; as de análise de tendências receberam a menor nota (1), por serem as mais demoradas.

Tabela 3

Avaliação por Família no Critério Tempo Necessário para Obter o Resultado

\begin{tabular}{cccccccccc}
\hline Critérios & $\begin{array}{c}\text { Opinião } \\
\text { especial. }\end{array}$ & Cenários & $\begin{array}{c}\text { Análise } \\
\text { tend. }\end{array}$ & $\begin{array}{c}\text { Aval. e } \\
\text { decisão }\end{array}$ & $\begin{array}{c}\text { Model. e } \\
\text { simul. }\end{array}$ & Criatividade & $\begin{array}{c}\text { Descrit. } \\
\text { matrizes }\end{array}$ & Estatísticas & $\begin{array}{c}\text { Monit. e } \\
\text { sistemas }\end{array}$ \\
\hline Tempo & 4 & 2 & 1 & 4 & 3 & 5 & 3 & 3 & 3 \\
\hline
\end{tabular}

Nota. Fonte: elaborada pelos autores.

\section{Critério: complexidade da operacionalização}

Na família de opinião de especialistas, Pesquisas de Opinião são relativamente fáceis de serem feitas, entendidas pelos pesquisados e utilizadas pelos tomadores de decisão. Mas os processos de grande escala, como Charrette e Syncon, mostram-se mais complexos pela necessidade do uso de equipamentos 
tais como sistemas de televisão e de transmissão. As pessoas também podem não ter disponibilidade de viajar para um local e lá ficar por vários dias (Glenn, 2003b).

Na família de cenários, a técnica FAR é relativamente fácil de explicar, mas difícil de aplicar. FAR é um processo de quatro etapas e cíclico, pois os cenários desenvolvidos na etapa 4 irão fornecer os insumos necessários para a etapa 1 de um ciclo subsequente, levando a melhores cenários (Coyle, 2003). Já de acordo com Glenn (2003c), a flexibilidade na estrutura analítica da técnica de Cenários permite que os planejadores entendam melhor as necessidades alternativas dos anos vindouros. Isso reduz significativamente a necessidade de previsões específicas, simplificando o processo.

Nas técnicas de análise de tendências, TIA é uma técnica de previsão bastante potente, ainda que relativamente simples e fácil de usar. Esta técnica aumenta a precisão das abordagens para a extrapolação de tendências (Gordon, 2003a). São necessários dados históricos para montagem da curva inicial, pareceres de especialistas em relação aos eventos inesperados, e um computador para processar as informações.

No tocante à complexidade da operacionalização das técnicas de avaliação e decisão, o AHP é simples, intuitivamente natural, fácil para o pensamento em geral e não requer especialização para entender e dominar (Saaty, 2008). Já a Análise de Decisão é tipicamente um processo iterativo. Uma vez que o modelo foi construído, realiza-se a análise de sensibilidade, que responde a perguntas condicionais. Em geral são necessárias várias iterações antes de ser encontrada uma solução satisfatória (Clemen, 1996). No caso da Árvore de Relevância, as entradas em um nível, em conjunto, destinam-se a descrever completamente o item ao qual se encontram ligadas no nível acima. Mas esta condição é muitas vezes difícil de conseguir na prática. Existem programas de computador que facilitam o processo (The Futures Group International, 2003).

Nas técnicas de modelagem e simulação, com a disponibilidade de computadores de alta velocidade, a Modelagem de Agentes tem sido cada vez mais aplicada a situações complexas. Engenheiros podem criar milhões de variantes e testá-las para encontrar a que apresenta o melhor desempenho (Gordon, 2003b). Estudos de Cross-Impact focalizam as interações entre pares de eventos. No mundo real, as interações importantes podem envolver não só pares, mas trios, ou mais. No entanto, se tais interações forem incluídas, a complexidade do julgamento crescerá (Gordon, 2003c). Simulações de futuro são projetos complexos. Um extenso trabalho preparatório deve ser feito antes, e os computadores podem contribuir imensamente para facilitar a avaliação dos resultados (Rausch \& Catanzaro, 2003). Transformar a simulação em um jogo requer recursos. A complexidade do modelo e o programa para manipulá-lo dependem do número de variáveis e de suas respectivas relações (Rausch \& Catanzaro, 2003).

No que concerne à complexidade da operacionalização, observa-se que algumas técnicas de criatividade são mais complexas, como TRIZ e Vision Generation, e outras menos, como Brainstorming. Dessa forma, os propósitos da pesquisa devem ser adequados à complexidade do uso. Analisando a família de descritivas e matrizes, pode-se afirmar que são técnicas complexas que partem do princípio de que os padrões de adesão a novas tecnologias pelo mercado e de desenvolvimento tecnológico são análogos aos do passado. Aplicando estas técnicas, é possível identificar as afinidades adequadas e analisar as semelhanças e diferenças (CGEE, n.d.).

Nas técnicas estatísticas, o pesquisador enfrentará uma etapa de coleta de dados, que deverá ter lógica e coerência para que, na segunda etapa (análise estatística), seus resultados possam ser confiáveis ao serem analisados. Dessa forma, o grau de complexidade é alto.

As técnicas da família de monitoramento e sistemas de inteligência são relativamente complexas e podem ser utilizadas para auxiliar em outras técnicas prospectivas, como a de cenários, que tem por finalidade vislumbrar possíveis conjunturas vindouras. Em relação à Análise Bibliométrica, o objetivo é quantificar os processos de informação escrita em revistas, patentes, artigos científicos, etc. A técnica ajuda a identificar padrões não aparentes, classificando as informações por autores, organizações, países, colaborações, cocitações, e assim por diante, utilizando procedimentos tais como 
análise fatorial e de cluster, mapas conceituais, análise de citação e de cocitação, entre outros (Daim et al., 2006). Devido à multiplicação dos bancos de dados que compilam informações e do poder de processamento dos computadores, o uso da Análise Bibliométrica enriquece uma base empírica de pesquisa (Huang, Zhang, Guo, Zhu, \& Porter, 2014).

A partir da análise das famílias, foram atribuídas as seguintes notas neste critério, apresentadas na Tabela 4: as técnicas de análise de tendências, apesar de apresentarem alguma complexidade, receberam nota 4 por serem mais simples quando comparadas às outras famílias; em seguida, criatividade, opinião de especialistas e avaliação e decisão receberam notas 3; as técnicas de modelagem e simulação receberam as menores notas (1) devido à sua maior complexidade; as demais famílias receberam nota 2 .

Tabela 4

\section{Avaliação por Família no Critério Complexidade da Operacionalização}

\begin{tabular}{cccccccccc}
\hline Critérios & $\begin{array}{c}\text { Opinião } \\
\text { especial. }\end{array}$ & Cenários & $\begin{array}{c}\text { Análise } \\
\text { tend. }\end{array}$ & $\begin{array}{c}\text { Aval. e Model. } \\
\text { decisão e simul. }\end{array}$ & Criatividade & $\begin{array}{c}\text { Descrit. } \\
\text { matrizes }\end{array}$ & Estatísticas & $\begin{array}{c}\text { Monit. e } \\
\text { sistemas }\end{array}$ \\
\hline Complexidade & 3 & 2 & 4 & 3 & 1 & 3 & 2 & 2 & 2 \\
\hline
\end{tabular}

Nota. Fonte: elaborada pelos autores.

\section{Critério: tipo de análise (subjetiva ou objetiva)}

Na família de opinião de especialistas, no tocante ao tipo de análise (subjetiva ou objetiva), os resultados dessas técnicas tendem a ser mais normativos (o que o futuro deveria ser), do que analíticos (o que o futuro pode ser). Tais processos não são tão bons para fazer planos específicos. Técnicas mais analíticas devem ser usadas de forma complementar (Glenn, 2003b).

Nas técnicas de cenários, a técnica FAR é fundamentada em imaginação, percepção e julgamento, portanto, é mais subjetiva. Por outro lado, permite que as trajetórias lógicas que geraram os cenários produzidos sejam facilmente rastreadas (Coyle, 2003). A geração de Cenários é também em grande parte uma técnica qualitativa. Cenários devem ser julgados pela capacidade de ajudar os decisores a elaborar políticas agora, em vez de apontar se no futuro estarão certos ou errados (Glenn, 2003c).

Nas técnicas de análise de tendências, no processo de Precursor Analysis é gerado um conjunto de condições anômalas consideradas dignas de uma avaliação mais profunda. Para cada condição, atribui-se uma específica métrica de risco de produzir graves consequências, chamada de índice de problema potencial (PPI). Na etapa de classificação, uma pontuação numérica de risco é calculada com base em índices de consequência e níveis de aplicabilidade (NASA, 2011), gerando resultados bastante objetivos.

No TIA, julgamento e imaginação são fundamentais para a implementação, principalmente na preparação da lista de eventos potenciais. Esses eventos devem ser plausíveis, impactantes e verificáveis em retrospecto. O programa de computador combina os eventos potenciais com os resultados da extrapolação original para produzir uma extrapolação ajustada (Gordon, 2003a), gerando resultados bem objetivos.

Em relação às técnicas de avaliação e decisão, a AHP incorpora julgamentos e valores pessoais de uma forma lógica. A técnica depende de imaginação, experiência e conhecimentos para compor a hierarquia de um problema, e de lógica, intuição e experiência para fornecer julgamentos (Saaty, 2008). A Análise de Decisão fornece uma forma estruturada de pensar sobre decisões, e também de envolver crenças e sentimentos. Portanto, julgamentos subjetivos desempenham papel central (Clemen, 1996). A técnica Árvores de Relevância permite uma análise sistemática da estrutura atual e futura de uma indústria (ou sistema) e a identificação de lacunas importantes (The Futures Group International, 2003). 
Tendo em consideração a família de modelagem e simulação, na Cross-impact analysis, testes de políticas são realizados pela definição de uma ação que possa afetar os eventos na matriz. Quando mudanças inesperadas acontecem, elas podem ser rastreadas de modo que as cadeias de causalidade sejam determinadas, e os efeitos da política, entendidos. Dessa forma, a matriz de impacto cruzado torna-se um modelo para apresentar os efeitos de impactos causados por ações políticas (Gordon, 2003c), tornando os resultados objetivos. Quanto ao tipo de análise (subjetiva ou objetiva), as técnicas de criatividade estão focadas em gerar dados brutos e subjetivos a serem lapidados posteriormente no processo de previsão.

Por trabalhar com manipulação de dados, em grande parte quantitativos, as técnicas descritivas e matrizes apresentam-se como objetivas. O mesmo ocorre com as técnicas estatísticas: em se tratando de análise quantitativa de dados, os produtos resultantes são bem objetivos. A maioria das técnicas de monitoramento e sistemas de inteligência é baseada na análise das publicações e da quantidade em que são observados os termos da pesquisa em tais publicações. Dessa forma, a Análise Bibliométrica pode ser considerada objetiva, mas a Análise de Conteúdo é mais subjetiva.

A partir da análise das famílias, foram atribuídas as seguintes notas neste critério, apresentadas na Tabela 5: as técnicas de análise de tendências, descritivas e matrizes e estatísticas receberam a maior nota (5) por serem as mais objetivas, o que facilita a interpretação e a aplicação prática dos resultados; em seguida, as de modelagem e simulação receberam nota 4; monitoramento e sistemas, nota 3; cenários, avaliação e decisão e opinião de especialistas, nota 2; criatividade, nota 1, por ser a mais subjetiva.

Tabela 5

Avaliação por Família no Critério Tipo de Análise (Subjetiva ou Objetiva)

\begin{tabular}{cccccccccc}
\hline Critérios & $\begin{array}{c}\text { Opinião } \\
\text { especial. }\end{array}$ & Cenários & $\begin{array}{c}\text { Análise } \\
\text { tend. }\end{array}$ & $\begin{array}{c}\text { Aval. e } \\
\text { decisão }\end{array}$ & $\begin{array}{c}\text { Model. } \\
\text { e simul. }\end{array}$ & Criatividade & $\begin{array}{c}\text { Descrit. } \\
\text { matrizes }\end{array}$ & Estatísticas & $\begin{array}{c}\text { Monit. e } \\
\text { sistemas }\end{array}$ \\
\hline Tipo Análise & 2 & 2 & 5 & 2 & 4 & 1 & 5 & 5 & 3 \\
\hline
\end{tabular}

Nota. Fonte: elaborada pelos autores.

\section{Critério: confiabilidade dos resultados}

Na família de opinião de especialistas, quanto à confiabilidade dos resultados, processos que envolvem grandes grupos podem transformar propostas avançadas em outras menos precisas de modo a serem aceitáveis para todos. Também podem resultar em uma análise superficial e não isenta por aqueles que sabem como manipular a técnica. Por outro lado, processos democráticos costumam resultar em um produto mais equitativo devido à partilha de ideias e valores pelos participantes durante a execução (Glenn, 2003b).

A boa facilitação de uma técnica participativa exige que o facilitador mantenha uma clara distinção entre as questões do processo e as questões do conteúdo. Confundir estes tópicos torna o recurso menos eficiente, e o conteúdo, menos profundo, diminuindo a confiança na integridade do resultado (Glenn, 2003b). Especificamente em relação ao Delphi, esta é uma técnica poderosa quando usada para procurar respostas a perguntas adequadas. Mas muita atenção deve ser dada à escolha dos participantes, e os questionários devem ser meticulosamente preparados e testados para evitar ambiguidade (Gordon, 2003d).

Em referência à confiabilidade dos resultados das técnicas de cenários, no FAR todas as opções são examinadas antes que as inviáveis sejam descartadas, e a técnica gera tantos cenários quantos necessários. Mas, paradoxalmente, o fato de usar a intuição e a criatividade é percebido como uma fraqueza (Coyle, 2003). A questão é que estudos futuros, e técnicas de cenário em particular, são fundamentalmente atos de imaginação com base em evidências (Coyle, 2003). Por outro lado, Cenários são uma das maneiras mais fáceis de apresentar informações complexas para os tomadores de decisão, tornando as possibilidades futuras mais reais (Glenn, 2003c). 
Nas técnicas de análise de tendências, embora na Precursor Analysis a classificação das anomalias empregue análise qualitativa, os racionais utilizados para atribuir o PPI deverão ser fundamentados em provas sólidas (NASA, 2011). A técnica TIA é usada para adicionar quantificação a um cenário, ajudando a garantir a consistência interna. Por outro lado, a lista de eventos potenciais dificilmente se mostra completa, e as probabilidades e os julgamentos são baseados na percepção de especialistas, podendo não ser precisos (Gordon, 2003a).

Na família de avaliação e decisão, o AHP pode ser aplicado a problemas reais e é particularmente útil para alocação de recursos, planejamento, e resolução de conflitos. Ele fornece uma escala para medir tópicos intangíveis e um método para estabelecer prioridades (Saaty, 2008). Em geral, a Análise de Decisão consiste em um modelo e em técnicas para lidar com decisões difíceis, e a incorporação de julgamentos subjetivos representa um aspecto importante (Clemen, 1996). A análise de Árvores de Relevância pode render uma superabundância de possibilidades. O julgamento humano ainda é necessário para direcionar o resultado, e, portanto, a técnica está sujeita a erro (The Futures Group International, 2003).

Quanto à confiabilidade da família de modelagem e simulação, na Modelagem de Agentes, apesar da simplicidade das regras, os resultados são complexos, e não é possível prever o comportamento do modelo (Gordon, 2003b). O Cross-Impact assume que, em certas aplicações, as probabilidades condicionais são mais precisas do que as estimativas a priori, o que não foi provado (Gordon, 2003c). Simulação e jogos chamam a atenção sobre as possíveis mudanças que podem ocorrer em um cenário existente ou imaginário e exploram as suas repercussões, mas não oferecem previsões, nem mesmo fornecem probabilidades de ocorrência sem o uso de outras técnicas (Rausch \& Catanzaro, 2003).

Em relação às técnicas de criatividade, sendo técnicas que estimulam a geração de ideias, a imaginação e a originalidade, é recomendado que sejam usadas no início de um processo para aumentar a habilidade de visualizar futuros alternativos (CGEE, n.d.). As técnicas descritivas e matrizes são consideradas confiáveis por se tratar de modelos de comparação, mapas e matrizes, que possuem resultados quantificáveis. Da mesma forma, as técnicas estatísticas são bem confiáveis, desde que o problema seja bem delineado, pois estas técnicas buscam mensurar o impacto de uma ou mais variáveis independentes no desempenho futuro de uma variável dependente, de forma numérica e precisa (CGEE, n.d.).

Em relação às técnicas de monitoramento e sistemas de inteligência, alguns pesquisadores relatam que há muita chance de a Bibliometria, bem como de outras técnicas desta família, crescer em uso, uma vez que evoluem as tecnologias que quantificam os processos de informações escritas (Coates et al., 2001). Neste sentido, os resultados são relativamente confiáveis.

A partir da análise das famílias, foram atribuídas as seguintes notas neste critério, apresentadas na Tabela 6: as técnicas estatísticas receberam a maior nota (5) por serem mais confiáveis, tanto na replicabilidade quanto na constância dos resultados; em seguida, descritivas e matrizes e monitoramento e sistemas receberam nota 4; as técnicas de criatividade, nota 2, por potencialmente apresentarem resultados mais variáveis, dependendo da dinâmica da atividade e das pessoas envolvidas; as demais famílias receberam nota 3 .

Tabela 6

Avaliação por Família no Critério Confiabilidade dos Resultados

\begin{tabular}{cccccccccc}
\hline Critérios & $\begin{array}{c}\text { Opinião } \\
\text { especial. }\end{array}$ & Cenários Anális Aval. e $\begin{array}{c}\text { Model. } \\
\text { e tend. decisão }\end{array}$ & Criatividade & $\begin{array}{c}\text { Descrit. } \\
\text { matrizes }\end{array}$ & Estatísticas & $\begin{array}{c}\text { Monit. e } \\
\text { sistemas }\end{array}$ \\
\hline Confiabilidade & 3 & 3 & 3 & 3 & 3 & 2 & 4 & 5 & 4 \\
\hline
\end{tabular}

Nota. Fonte: elaborada pelos autores. 


\section{Tabela comparativa e análise dos resultados}

Com base nas avaliações feitas por família em relação aos critérios definidos, foi elaborada uma tabela comparativa (Tabela 7) com o objetivo de fazer um resumo visual das famílias de técnicas no tocante a cada critério.

Tabela 7

Avaliação das Técnicas por Família e por Critérios

\begin{tabular}{lccccccccc}
\hline Critérios & $\begin{array}{c}\text { Opinião } \\
\text { especial. }\end{array}$ & $\begin{array}{c}\text { Cenários } \\
\text { Análise } \\
\text { tend. }\end{array}$ & $\begin{array}{c}\text { Aval. e } \\
\text { decisão e simul. }\end{array}$ & Criatividade & $\begin{array}{c}\text { Descrit. } \\
\text { matrizes }\end{array}$ & $\begin{array}{c}\text { Estatísticas } \\
\text { Monit. e } \\
\text { sistemas }\end{array}$ \\
\hline Núm. pessoas & 2 & 2 & 4 & 5 & 2 & 3 & 2 & 2 & 1 \\
Tempo & 3 & 3 & 3 & 5 & 3 & 3 & 2 & 2 & 2 \\
Complexidade & 4 & 2 & 1 & 4 & 3 & 5 & 3 & 3 & 3 \\
Tipo Análise & 2 & 2 & 4 & 3 & 1 & 3 & 2 & 2 & 2 \\
Confiabilidade & 3 & 2 & 5 & 2 & 4 & 1 & 5 & 5 & 3 \\
\hline
\end{tabular}

Nota. Fonte: elaborada pelos autores.

Em relação ao critério de análise custo para operacionalização, a família das técnicas de avaliação e decisão é a que apresenta os menores custos comparados com as demais, seguida de análise de tendências. Já as técnicas de monitoramento e sistemas apresentam os maiores custos. As demais famílias possuem custos relativamente similares. No critério número de pessoas, a família avaliação e decisão é a que necessita de menos pessoas. As demais são praticamente equivalentes neste critério.

Quanto ao tempo necessário, as técnicas de criatividade se sobressaem como as que necessitam de menos tempo para o processo e a obtenção dos resultados, seguidas pelas famílias avaliação e decisão e opinião de especialistas. As demais necessitam de mais tempo, com destaque para as técnicas de análise de tendências, que são as mais delongadas. No tocante à complexidade da operacionalização, a família de análise de tendências é a mais simples, e a família modelagem e simulação, a mais complexa.

No critério tipo de análise (subjetiva ou objetiva), as famílias das técnicas estatísticas, descritivas e matrizes e análise de tendências são as mais objetivas, enquanto a família criatividade é a mais subjetiva. Por fim, em confiabilidade dos resultados, a família das técnicas estatísticas é a mais confiável, seguida pelas famílias descritivas e matrizes e monitoramento e sistemas. As demais são semelhantes em termos de confiabilidade.

\section{Considerações Finais}

A partir dos estudos das técnicas e da comparação entre as famílias, observou-se que as notas das técnicas variam de acordo com os critérios de avaliação. Não é possível considerar uma família como a melhor, pois todas apresentam vantagens e desvantagens, dependendo do critério utilizado. Nesse sentido, é possível deduzir que a utilização de técnicas combinadas seja uma medida vantajosa para os interessados em estudos de prospecção, pois permite que as desvantagens de uma técnica sejam compensadas pelas vantagens de outra.

Diversos especialistas, como Armstrong (2001), Popper e Korte (2004), e Robinson, Huang, Guo e Porter (2013), concordam que é proveitoso usar várias técnicas simultaneamente, uma vez que cada uma só lida com aspectos limitados de previsão. A utilização combinada proporciona muitas vezes mais 
eficiência e faz com que as previsões sejam mais robustas. Armstrong (2001) analisa 57 estudos, de diferentes pesquisadores, que contribuíram para formar os princípios da combinação de técnicas. Segundo o autor, combinar técnicas de previsão é uma estratégia vantajosa. Em vez de tentar escolher a melhor, é mais interessante procurar quais técnicas ajudam a aumentar a precisão, assumindo que cada uma tem algo a contribuir (Armstrong, 2001). Já Popper e Korte (2004) observam que o uso de várias abordagens ajuda a processar as informações de diversas maneiras diferentes. Por outro lado, o tempo é uma restrição que obriga a equipe de prospecção a conduzir a análise dos resultados e a elaboração de relatórios parciais e finais em menos tempo.

Robinson et al. (2013), ao apresentarem os requisitos do seu modelo teórico de prospecção, defendem que uma abordagem flexível é necessária para ciências e tecnologias emergentes, nas quais frequentemente os dados possíveis de serem obtidos diferem muito. Esses autores constatam uma combinação produtiva de técnicas qualitativas e quantitativas e o uso de uma variedade de ferramentas e abordagens no processo de prospecção dos rumos da inovação em ciências e tecnologias emergentes.

Popper (2008) levantou 886 estudos de prospecção de diversos países, de forma a analisar práticas prospectivas utilizadas pelo mundo, para debater a questão sobre como as técnicas são selecionadas. $\mathrm{O}$ presente trabalho, em vez de levantar como são escolhidas ou combinadas as técnicas de prospecção pelas empresas, visa a fornecer, como principal contribuição, um referencial de parâmetros para ajudar as empresas na seleção das técnicas mais adequadas, de acordo com as suas capacidades e necessidades específicas. Considerando os critérios apresentados e a avaliação de cada família, as organizações poderão embasar as suas escolhas de técnicas prospectivas. Outro ponto importante é o fato de a qualidade das previsões depender da seleção apropriada e da aplicação correta da técnica.

As principais limitações do estudo estão relacionadas à seleção dos critérios, e ao julgamento empregado para avaliar as famílias de técnicas (que se baseou nas características recolhidas na análise bibliográfica). Por isso se espera que este artigo sirva para iniciar uma discussão sobre os critérios e os valores atribuídos às técnicas, suscitando novas propostas de avaliação pela comunidade científica. A seleção de outros critérios e o uso de outras referências bibliográficas resultarão em análises comparativas diferentes, enriquecendo o processo. Como indicação para pesquisas futuras, sugere-se analisar as famílias em relação às médias ou às variações em torno da média dos critérios estabelecidos, e a elaboração de indicadores de desempenho com base nas notas atribuídas, facilitando a escolha da técnica mais apropriada.

Esta é uma área de estudo em constante evolução. É natural imaginar futuros alternativos e como suas ações podem modificá-los. Percebe-se que as recentes inovações tecnológicas e o crescimento das redes de comunicação têm causado grande impacto. $\mathrm{O}$ desenvolvimento de técnicas analíticas mais robustas ajuda o processo de avaliação e melhora a tomada de decisões nos diversos âmbitos da vida em sociedade.

\section{Referências}

Armstrong, J. S. (2001). Combining forecasts. In J. S. Armstrong (Ed.), Principles of forecasting: a handbook for researchers and practitioners (pp. 417-439). Norwell, MA: Kluwer Academic Publishers.

Battistella, C., \& De Toni, A. F. (2011). A methodology of technological foresight: a proposal and field study. Technological Forecasting \& Social Change, 78(6), 1029-1048. doi: 10.1016/j.techfore.2011.01.006

Carbone, R., \& Armstrong, J. S. (1982). Note. Evaluation of extrapolative forecasting methods: results of a survey of academicians and practitioners. Journal of Forecasting, 1(2), 215-217. doi: 10.1002/for.3980010207 
Centro de Gestão e Estudos Estratégicos. (n.d.). Prospecção em CT\&I. Recuperado de http://www.cgee.org.br/prospeccao

Clemen, R. T. (1996). Making hard decisions: an introduction to decision analysis (2nd ed.). Pacific Grove, CA: Duxbury Press.

Coates, V., Farooque, M., Klavans, R., Lapid, K., Linstone, H. A., Pistorius, C., \& Porter, A. L. (2001). On the future of technological forecasting. Technological Forecasting and Social Change, 67(1), 1-17. doi: 10.1016/S0040-1625(00)00122-0

Corbetta, P. (2003). Social research: theory, methods and techniques. London: Sage.

Coyle, R. G. (2003). Morphological forecasting - field anomaly relaxation (FAR). In J. C. Glenn \& T. J. Gordon (Eds.), Futures research methodology (V2.0, Chap. 19, pp. 1-22, CD-ROM). Washington, DC: American Council for the United Nations University.

Daim, T. U., Rueda, G., Martin, H., \& Gerdsri, P. (2006). Forecasting emerging technologies: use of bibliometrics and patent analysis. Technological Forecasting \& Social Change, 73(8), 981-1012. doi: $10.1016 / j$.techfore.2006.04.004

Dalkey, N., \& Helmer, O. (1963). An experimental application of the Delphi method to the use of experts. Management Science, 9(3), 458-467. doi: 10.1287/mnsc.9.3.458

Drucker, P. (1996). Administrando em tempos de grandes mudanças (3a ed.). São Paulo: Pioneira.

Glenn, J. C. (2003a). Introduction to the futures research methods series. In J. C. Glenn \& T. J. Gordon (Eds.), Futures research methodology (V2.0, Chap. 1, pp. 1-61, CD-ROM). Washington, DC: American Council for the United Nations University.

Glenn, J. C. (2003b). Participatory methods. In J. C. Glenn \& T. J. Gordon (Eds.), Futures research methodology (V2.0, Chap. 14, pp. 1-32, CD-ROM). Washington, DC: American Council for the United Nations University.

Glenn, J. C. (2003c). Scenarios. In J. C. Glenn \& T. J. Gordon (Eds.), Futures research methodology (V2.0, Chap. 13, pp. 1-25, CD-ROM). Washington, DC: American Council for the United Nations University.

Gordon, T. J. (2003b). Agent modeling. In J. C. Glenn \& T. J. Gordon (Eds.), Futures research methodology (V2.0, Chap. 21, pp. 1-35, CD-ROM). Washington, DC: American Council for the United Nations University.

Gordon, T. J. (2003c). Cross-impact analysis. In J. C. Glenn \& T. J. Gordon (Eds.), Futures research methodology (V2.0, Chap. 6, pp. 1-19, CD-ROM). Washington, DC: American Council for the United Nations University.

Gordon, T. J. (2003d). The Delphi method. In J. C. Glenn \& T. J. Gordon (Eds.), Futures research methodology (V2.0, Chap. 3, pp. 1-30, CD-ROM). Washington, DC: American Council for the United Nations University.

Gordon, T. J. (2003a). Trend impact analysis. In J. C. Glenn \& T. J. Gordon (Eds.), Futures research methodology (V2.0, Chap. 5, pp. 1-21, CD-ROM). Washington, DC: American Council for the United Nations University.

Hammersley, M. (1992). What is wrong with ethnography: methodological explorations. London: Rutledge. 
Huang, L., Zhang, Y., Guo, Y., Zhu, D., \& Porter, A. L. (2014). Four dimensional science and technology planning: a new approach based on bibliometrics and technology roadmapping. Technological Forecasting \& Social Change, 81(1), 39-48. doi: 10.1016/j.techfore.2012.09.010

Kirk, J., \& Miller, M. L. (1986). Reliability and validity in qualitative research. London: Sage.

Kotler, P., \& Keller, K. L. (2006). Administração de marketing (12a ed.). São Paulo: Pearson Prentice Hall.

Linstone, H. A. (2002). Corporate planning, forecasting and the long wave. Futures, 34(3/4), 317-336. doi: 10.1016/S0016-3287(01)00047-7

Linstone, H. A. (2003). The multiple perspective concept. In J. C. Glenn \& T. J. Gordon (Eds.), Future research methodology (V2.0, Chap. 24, pp. 1-19, CD-ROM). Washington, DC: American Council for the United Nations University.

Miles, I., \& Keenan, M. (2003, December). Overview of methods used in foresight. Proceedings of the Technology Foresight for Organizers Training Course, Ankara, Turkey.

Miles, I., Keenan, M., \& Kaivo-Oja, J. (2002). Handbook of knowledge society foresight. Dublin: Eurofound.

National Aeronautics and Space Administration. (2011, December). Accident precursor analysis handbook. Version 1.0. Retrieved from http://www.hq.nasa.gov/office/codeq/doctree/NASA_SP2011-3423.pdf

Popper, R. (2008). How are foresight methods selected? Foresight, 10(6), 62-89. doi: $10.1108 / 14636680810918586$

Popper, R., \& Korte, W. (2004, May). Xtreme euforia: combining foresight methods. Proceedings of the EU-US Scientific Seminar: New Technology Foresight, Forecasting \& Assessment Methods, Seville, Spain, 1.

Porter, A. L., Ashton, W. B., Clar, G., Coates, J. F., Cuhls, K., Cunningham, S. W., Ducatel, K., Duin, P. van der, Georgehiou, L., Gordon, T., Linstone, H., Marchau, V., Massari, G., Miles, I., Mogee, M., Salo, A., Scapolo, F., Smits, R., \& Thissen, W. (2004). Technology futures analysis: toward integration of the field and new methods. Technological Forecasting \& Social Change, 71(3), 287-303. doi:10.1016/j.techfore.2003.11.004

Rausch, E., \& Catanzaro, F. (2003). Simulation and games in futuring and other uses. In J. C. Glenn \& T. J. Gordon (Eds.), Futures research methodology (V2.0, Chap. 15, pp. 1-51, CD-ROM). Washington, DC: American Council for the United Nations University.

Reis, D. R., \& Lobo, R. C. G. (2015). Technological forecasting: the methodology used by a federation of industries in Brazil. Australian Journal of Basic and Applied Sciences, 9(20), 503-509.

Richardson, R. (1999). Pesquisa social: métodos e técnicas (3a ed.). São Paulo: Atlas.

Robinson, D. K. R., Huang, L., Guo, Y., \& Porter, A. L. (2013). Forecasting innovation pathways (FIP) for new and emerging science and technologies. Technological Forecasting \& Social Change, 80(2), 267-285. doi: 10.1016/j.techfore.2011.06.004

Roper, A. T., Cunningham, S. W., Porter, A. L., Mason, T. W., Rossini, F. A., \& Banks, J. (2011). Forecasting and management of technology (2nd ed.). New Jersey: John Wiley \& Sons, Inc.

Saaty, T. L. (2008). Decision making for leaders: the analytic hierarchy process for decisions in a complex world. Pittsburgh: RWS Publications. 
Silverman, D. (2006). Interpreting qualitative data (3rd ed.). London: Sage.

Sterman, J. D. (1985). The economic long wave: theory and evidence [Working Paper $\mathrm{n}^{\mathrm{o}}$ 1656-85]. Alfred P. Sloan School of Management, Massachusetts Institute of Technology. Cambridge, MA, U.S.A.

The Futures Group International. (2003). Relevance tree and morphological analysis. In J. C. Glenn \& T. J. Gordon (Eds.), Futures research methodology (V2.0, Chap. 12, pp. 1-13, CD-ROM). Washington, DC: American Council for the United Nations University.

\section{Dados dos Autores}

Dálcio Roberto dos Reis Rua Professor Pedro Viriato Parigot de Souza, 5300, Cidade Industrial, 81280-330, Curitiba, PR, Brasil. E-mail: dalcio.reis@gmail.com

Ticiana Braga de Vincenzi

Rua Professor Pedro Viriato Parigot de Souza, 5300, Cidade Industrial, 81280-330, Curitiba, PR, Brasil. E-mail: ticivin@hotmail.com

Fabricio Palermo Pupo

Rua Professor Pedro Viriato Parigot de Souza, 5300, Cidade Industrial, 81280-330, Curitiba, PR, Brasil. E-mail: fabriciopupo@hotmail.com 